\title{
Perceived sources of stress amongst Indian Dental Students in Bareilly City
}

\begin{abstract}
:
Introduction: In addition to the stresses pertaining to dentistry as a profession, dental students have to face the additional stress of their studies. Through stress can also contribute to decreased student performance. The aim of this study was to assess perceived sources of stress among undergraduate dentists at a Dental College in Bareilly, India.

Materials and methods: A modified dental environment stress (DES) questionnaire which consisted of 38 questions was used to assess the levels of stress.

Results: The first major stressor for all the students was examination and grades followed by full working day, fear of unemployment after graduation, receiving criticism from supervisors about academic or clinical work, amount of cheating in dental faculty, rules and regulations of the faculty. Fear of unemployment after graduation and lack of relaxation were among the six highest stressors in each year, at least four. The perception of stress between genders with a male predilection was significantly different. Eighteen of the 38 questionnaire items had significant differences across the year groups including clinical DES items.

Conclusion: The primary sources of stress perceived by dental students were exams and grades followed by full working day and receiving criticism about academic or clinical work from superiors. Since high levels of perceived stress can reduce student performance, dental schools should consider courses in stress management.
\end{abstract}

Keywords: Perceived stress, Dental undergraduates, India.

\section{Introduction:}

Stress as 'a stimulus, a response or the outcome of an interaction between the two, with the interaction represented as some imbalance between the individual and the environment.[1] Stress may be physiological only if it persists below a certain point, above which it continues to a degree of distress. Occupational stress can be characterized as a state of physical and mental distress caused by excessive demands or lack of resources.[2] In chronic or extreme circumstances occupational stress in the susceptible practitioner can precipitate a 'burnout' state.

Burnout is a state of emotional, physical, and mental exhaustion caused by excessive and prolonged stress. It occurs when you feel overwhelmed, emotionally drained, and unable to meet constant demands.

\begin{tabular}{|l|l|}
\hline \multicolumn{2}{|c|}{ Access this article online } \\
\hline \multirow{2}{*}{$\begin{array}{l}\text { Website: } \\
\text { www.ujds.in }\end{array}$} & Quick Response Code \\
\hline $\begin{array}{l}\text { DOI: } \\
\text { https://doi.org/10.21276/10.21276/ujds.2020.6.2.5 }\end{array}$ \\
\hline
\end{tabular}

Various studies showed high levels of stress and burnout among doctors and other psychological distress. 3 Psychological distresses is a general term used to describe

\author{
${ }^{1}$ SALEEM A, ${ }^{2}$ GUPTA $P,{ }^{3}$ SHIVALINGESH K K, \\ ${ }^{4}$ MIR H, ${ }^{5}$ SRIVASTAV D, ${ }^{6}$ SINGH A V \\ ${ }_{1,2,3,4,5}$ Institute of Dental Sciences, Bareilly. \\ ${ }^{6}$ Teerthanker Mahaveer Dental College and Research \\ Centre, Moradabad
}

Address for Correspondence : Dr. Adeeba Saleem Post Graduate, Institute of Dental Sciences, Bareilly-243006

Email : adeebasaleem2015@gmail.com

Received : 25 July 2020, Published : 31 August 2020

How to cite this article: Saleem, A., Gupta, P., K K, S., Mir, H., Srivastav, D., \& Singh, A. V. (2020). Perceived sources of stress amongst Indian dental students in Bareilly city. UNIVERSITY JOURNAL OF DENTAL SCIENCES, 6(2): 42-1. 
unpleasant feelings or emotions that impact your level of functioning. In other words, it is psychological discomfort that interferes with your activities of daily living. Psychological distress can result in negative views of the environment, others, and the self.

A study conducted by Basudan $\mathrm{S}$ et al, 4 among dental students found an association between different stress levels and mental health: high levels of stress were associated with depression, whereas low and moderate stress levels were associated with anxiety.

Dental school is also a highly stressed, demanding setting for the students.[5] The dental career was deemed the most demanding of all professions in health care.[2] Stress in dental students can be multifactorial, emerging from both the academic and socio-cultural environment and due to (emotional and financial) social support issues. Clinical student experiences are representative of those of qualified practitioners.[6] Apart from excellence in academic performance, the precise technical requirements of dentistry make the transition from preclinical to clinical all the more stressful.[7]

In the current study, the solution to stress problem in dental school is to identify perceived causes of stress that need a more in-depth investigation and to briefly consider some possible outcomes of perceived stress. In Bareilly, no studies on Perceived sources of stress amongst Indian dental students were available on search of literature. Thus, there is a need for an investigation focused on the assessment of perceived sources among undergraduate students.

\section{Aim And Objectives of Study:}

The aim of this research is to determine perceived sources of stress amongst undergraduate dental students in Dental College located in Bareilly, Uttar Pradesh, India.

\section{The objectives of this study are:}

1. To determine the different problems facing by Dental Environment Scale (DES) amongst dental students in the dental college of Bareilly city that may serve as possible stressors;

2. To investigate the role of gender, marital status, living environment and the academic year on problem perception;

3. To study the effect of the perceived problems upon the general academic performance of students.
Materials and Method:

A cross-sectional questionnaire study was conducted amongst undergraduate dental students in Dental College located in Bareilly, Uttar Pradesh, India for a period of 2 months from December 2019 to January 2020. The Dental Environment Stress questionnaire (DES) 8 was used to assess sources of perceived stress associated with undergraduate dental students.

Verbal informed consent was obtained, and the questionnaires were given at the individual level. Prior to commence of the study, an ethical clearance was obtained from the Institutional Ethical Committee of Institute of Dental Sciences, Bareilly.

Undergraduate dental education system in India is divided into two part; preclinical and clinical dentistry, where preclinical dentistry includes 1 st and 2nd year students whom have not had any clinical experience, and clinical dentistry constitutes of $3 \mathrm{rd}$, final year and interns. The study subjects were made fully aware about the nature of the study and its design.

The sample size calculated was 210 by using GPower 3.1 software, but considering the dropouts, a total of $214(\mathrm{~N}=214)$ participants were selected. The total students present on the day of questionnaire distribution were 67, 64, 52, 45 and 77 among 1st, 2nd, 3rd, final year and Interns respectively, that constituted a total of 305 undergraduate students. Simple random sampling method was used for selection of students. There were 108 clinical students ( $3 \mathrm{rd}$ year $=36$, final year $=36$ and interns $=36)$ and 106 non-clinical students $(1$ st year $=53$, 2nd year $=53$ ).

\begin{tabular}{|c|c|c|c|}
\hline \multirow[t]{4}{*}{ Input: } & Effect size f & $=$ & 0.25 \\
\hline & $\alpha$ err prob & $=$ & 0.05 \\
\hline & Power (1- $\beta$ err prob) & $=$ & 0.95 \\
\hline & Number of groups & $=$ & 2 \\
\hline \multirow[t]{6}{*}{ Output: } & Noncentrality parameter? & $=$ & 13.1250000 \\
\hline & Critical F & $=$ & 3.8865546 \\
\hline & Numerator df & $=$ & 1 \\
\hline & Denominator df & $=$ & 208 \\
\hline & Total sample size & $=$ & 210 \\
\hline & Actual power & $=$ & 0.9501287 \\
\hline
\end{tabular}

Stress was assessed using a modified dental environment stress (DES) questionnaire8, 38-item questionnaire based on a 4-point Likert scale, with scores ranging from 1 (not stressful) to 4 (very stressful) applicable to the Indian context in dental education. Clinical training questions $[3,4,10,16$, $18,19,25,26,27,28,29$ and 38] were excluded from the questionnaire granted to non-clinical students (first and 
second grades). All participants took part in the study voluntarily and no incentives were used for the respondents. The questionnaire was adapted from pre-tested and prevalidated questionnaires from previous studies.

\section{Statistical Analysis:}

In the next stage, the distributed and completed questionnaires were collected and the obtained data was entered in MS Excel sheet (windows 2007). Statistical analysis of the data was done using the statistical package for the social science (SPSS 22.0) using One way ANOVA to determine mean significant difference between the different variables, $\mathrm{p}<0.05$ consider statistically significant.

\section{Result:}

The overall response rate was $93.0 \%(214 / 230)$. The present study was conducted among 214 participants out of which 149 were females and 65 were males (Table 1). There was no significant difference in sex distribution of patients according to year of study $\left(* X^{2}\right.$-value $=6.2416$, $p$-value $\left.=0.183\right)$.

TABLE 1 - Sample distribution by year of study and gender.

\begin{tabular}{|l|l|l|l|l|l|l|}
\hline & \multicolumn{3}{|l|}{ Male } & \multicolumn{2}{l|}{ Female } & \multicolumn{2}{l|}{ Total } \\
\hline Year of study & Number & Percentage (\%) & Number & Percentage (\%) & Number & Percentage (\%) \\
\hline Ist year & 16 & 30.2 & 37 & 69.8 & 53 & 100.0 \\
\hline II year & 11 & 20.8 & 42 & 79.2 & 53 & 100.0 \\
\hline III year & 14 & 38.9 & 22 & 61.1 & 36 & 100.0 \\
\hline IV year & 9 & 25.0 & 27 & 75.0 & 36 & 100.0 \\
\hline Intern & 15 & 41.7 & 21 & 58.3 & 36 & 100.0 \\
\hline & 65 & $30.3 \%$ & 149 & $69.6 \%$ & 214 & 100.0 \\
\hline
\end{tabular}

$* \mathrm{X}^{2}$-Value $=6.2416, \mathrm{p}$-Value $=0.183$ (not significant)

There was no significant difference in sex distribution of patients according to year of study.

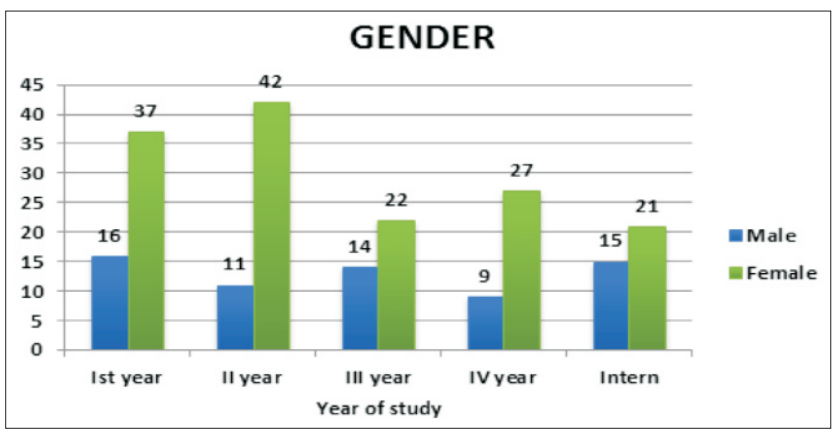

Fig. 1. Sample distribution by year of study and gender.

TABLE 2- Frequency and distribution by year of study with stay during your student years

\begin{tabular}{|l|l|l|l|l|l|l|}
\hline Stay During student Year & I year & II year & III year & IV year & Intern & \\
\hline In a hostel & 30 & 36 & 24 & 17 & 19 & $126(58.8 \%)$ \\
\hline In a flat/house without parents & 2 & 1 & 1 & 7 & 8 & $19(8.8 \%)$ \\
\hline In flat / house with parents & 21 & 16 & 10 & 11 & 9 & $67(31.3 \%)$ \\
\hline Others & 0 & 0 & 1 & 1 & 0 & $2(0.9 \%)$ \\
\hline
\end{tabular}

Table 2 Showing where do participants stay during their student years. The greatest proportions of students $58.8 \%$ were lived in hostel; $31.3 \%$ living in flats or living with parents or their own accommodation; less than $9 \%$ resided at in a flat/house without parents. The first and second year mean score for the individual stressor moving away from home throughout the course. There was no significant difference in year of study with stay during your student years.

TABLE 3- Frequency and distribution by year of study with watching TV on a daily basis (hours)

\begin{tabular}{|l|l|l|l|l|l|l|}
\hline Watch TV daily & I year & II year & III year & IV year & Intern & \\
\hline Less 0.5 hour & 41 & 41 & 30 & 26 & 20 & 140 \\
\hline $0.5-1$ hour & 6 & 5 & 3 & 5 & 7 & 26 \\
\hline $1-2$ hours & 4 & 4 & 2 & 2 & 4 & 16 \\
\hline 2-3 hours & 0 & 0 & 1 & 0 & 2 & 3 \\
\hline More than 3 hours & 2 & 3 & 0 & 3 & 3 & 11 \\
\hline
\end{tabular}

TABLE 3 Majority of students (140) watched TV for less than 30 minutes, followed by 26 students who watched TV for less than 30 minutes-1 hour. However there were no statistically significant differences present between the years of study with watching TV.

TABLE 4- Frequency and distribution by year of study and marital status

\begin{tabular}{|l|l|l|l|l|l|l|}
\hline Marital status & I year & II year & III year & IV year & Intern & \\
\hline Single & 50 & 50 & 33 & 33 & 31 & 197 \\
\hline Living with a spouse/partner & 1 & 0 & 1 & 1 & 1 & 4 \\
\hline Living with friends & 0 & 1 & 2 & 2 & 4 & 9 \\
\hline Others & 2 & 2 & 0 & 0 & 0 & 4 \\
\hline
\end{tabular}

TABLE 4 Almost all (197 students) were single, while as the rest of them (i.e. 17 students) were living with their friends/spouse.

TABLE 5- Frequency and distribution by year of study and hours used internet on a daily basis

\begin{tabular}{|l|l|l|l|l|l|l|}
\hline Use Internet daily & I year & II year & III year & IV year & Intern & \\
\hline $0.5-1$ hour & 11 & 10 & 4 & 5 & 5 & 26 \\
\hline $1-2$ hours & 18 & 16 & 7 & 11 & 8 & 60 \\
\hline $2-3$ hours & 13 & 12 & 14 & 7 & 7 & 53 \\
\hline & & & & & & \\
More than 3 hours & 11 & 15 & 11 & 13 & 16 & 66 \\
\hline
\end{tabular}

TABLE 5 Most than half of the participants (66 students) spent more than 3 hours on internet and those were mostly the interns (16).

TABLE 6- Frequency and distribution by year of study and lived during childhood and adolescence

\begin{tabular}{|l|l|l|l|l|l|l|}
\hline Live during childhood & I year & II year & II year & IV year & Intern & Total \\
\hline Urban area & 48 & 45 & 34 & 32 & 29 & 188 \\
\hline Rural area & 5 & 8 & 2 & 4 & 7 & 26 \\
\hline
\end{tabular}

TABLE 6 Furthermore the findings revealed that majority of the participants (188 students) were from the urban areas and the remaining 26 were from the rural background where as there was no significant difference the two. 
University J Dent Scie 2020; Vol. 6, Issue 2

TABLE 7- Perceived sources of stress, mean stress scores, standard deviation, and significant differences between years of study.

\begin{tabular}{|c|c|c|c|c|c|c|c|c|}
\hline Q.No. & Questions & I year & Il year & III year & IV year & Intern & F-VALUE & P-VALUE \\
\hline \multicolumn{9}{|c|}{ Self-efficacy beliefs } \\
\hline 1 & $\begin{array}{l}\text { Lack of confidence to be a successful dental } \\
\text { student }\end{array}$ & $1.77 \pm 0.78$ & $1.98 \pm 0.84$ & $2.19 \pm 0.82$ & $2.25 \pm 1$ & $2.22 \pm 0.93$ & $\begin{array}{l}2.518 \\
4,5,3>2,1 \\
\end{array}$ & $0.042^{*}$ \\
\hline 2 & Lack of confidence to be a successful dentist & $1.91 \pm 0.86$ & $2.02 \pm 0.89$ & $2.11 \pm 1.09$ & $2.42 \pm 1.11$ & $2.19 \pm 1.01$ & 1.646 & 0.164 \\
\hline 3 & Completing clinical requirements & & & $2.31 \pm 0.82$ & $2.42 \pm 1.08$ & $2.19 \pm 1.04$ & $\begin{array}{l}55.026 \\
4>3,5 \\
\end{array}$ & $0.000^{*}$ \\
\hline 4 & $\begin{array}{l}\text { Fear of not having possibility to pursue a post } \\
\text { graduate dental education programme }\end{array}$ & & & $2 \pm 0.93$ & $2.11 \pm 1.04$ & $2.14 \pm 1.2$ & $\begin{array}{l}68.690 \\
5,4>3\end{array}$ & $0.000^{*}$ \\
\hline 5 & Lack of confidence in own decision making & $1.74 \pm 0.74$ & $1.79 \pm 0.82$ & $2.03 \pm 1.06$ & $2.03 \pm 1.03$ & $1.97 \pm 0.94$ & 1.037 & 0.389 \\
\hline 6 & Fear of failing a course or a year & $1.94 \pm 1.01$ & $1.79 \pm 0.97$ & $2.47 \pm 1.11$ & $2.33 \pm 1.07$ & $2.11 \pm 1.28$ & $\begin{array}{l}2.857 \\
3,4>5>1,2\end{array}$ & $0.025^{*}$ \\
\hline 7 & Difficulty in understanding lecture materials & $1.83 \pm 0.85$ & $1.81 \pm 0.81$ & $1.72 \pm 0.81$ & $2.17 \pm 0.91$ & $1.72 \pm 0.61$ & 1.861 & 0.118 \\
\hline 8 & Language barrier & $1.42 \pm 0.72$ & $1.53 \pm 0.8$ & $1.5 \pm 0.94$ & $1.72 \pm 0.91$ & $1.5 \pm 0.81$ & 0.760 & 0.552 \\
\hline 9 & $\begin{array}{l}\text { Fear of unable to catch up if getting behind } \\
\text { the work }\end{array}$ & $2.04 \pm 0.92$ & $2.19 \pm 0.96$ & $1.92 \pm 0.77$ & $1.92 \pm 0.87$ & $1.83 \pm 0.77$ & 1.127 & 0.345 \\
\hline \multicolumn{9}{|c|}{ Faculty and administration } \\
\hline 10 & Atmosphere created by clinical supervisors & & & $2.25 \pm 0.87$ & $2.67 \pm 0.79$ & $2.56 \pm 1.05$ & 2.008 & 0.139 \\
\hline 11 & $\begin{array}{l}\text { Receiving criticism from supervisors about } \\
\text { academic or clinical work }\end{array}$ & $1.91 \pm 1.08$ & $1.72 \pm 0.82$ & $2.33 \pm 1.1$ & $2.56 \pm 0.88$ & $2.67 \pm 1.2$ & $\begin{array}{l}7.26 \\
5,4>3,1,2\end{array}$ & $0.000^{*}$ \\
\hline 12 & Amount of cheating in dental faculty & $1.45 \pm 0.85$ & $1.77 \pm 0.87$ & $2.36 \pm 1.17$ & $2.25 \pm 1.05$ & $2.17 \pm 1.18$ & $\begin{array}{l}6.330 \\
3>4>5>2,1\end{array}$ & $0.000^{*}$ \\
\hline 13 & Rules and regulations of the faculty & $1.92 \pm 1.25$ & $1.89 \pm 0.95$ & $2.5 \pm 1.06$ & $2.61 \pm 1.02$ & $2.53 \pm 1.11$ & $\begin{array}{l}4.693 \\
4>5>3>1>2 \\
\end{array}$ & $0.001^{*}$ \\
\hline 14 & Approachability of teaching staff & $1.64 \pm 1.04$ & $1.74 \pm 0.92$ & $1.89 \pm 0.98$ & $2.25 \pm 1.2$ & $1.94 \pm 0.89$ & 2.238 & 0.066 \\
\hline 15 & $\begin{array}{l}\text { Expectation of dental faculty and what in } \\
\text { reality it is like }\end{array}$ & $1.89 \pm 1.1$ & $1.77 \pm 0.89$ & $2.31 \pm 0.92$ & $2.25 \pm 0.94$ & $2.44 \pm 0.91$ & $\begin{array}{ll}3.936 \\
5>3,4>1,2 \\
\end{array}$ & $0.004^{*}$ \\
\hline 16 & Availability of supervisors in clinic & & & $2.08 \pm 0.81$ & $2.17 \pm 1.06$ & $2.03 \pm 1.13$ & 0.173 & 0.841 \\
\hline 17 & $\begin{array}{l}\text { Attitudes of faculty towards women dental } \\
\text { students }\end{array}$ & $1.6 \pm 1.06$ & $1.47 \pm 0.82$ & $1.56 \pm 0.84$ & $2.11 \pm 1.17$ & $2.03 \pm 1.11$ & $\begin{array}{l}3.463 \\
4,5>1,3,2 \\
\end{array}$ & $0.009^{*}$ \\
\hline 18 & Shortage of allocated clinical time & & & $2.22 \pm 0.96$ & $2.42 \pm 1.2$ & $2.03 \pm 1.11$ & 1.135 & 0.325 \\
\hline 19 & $\begin{array}{l}\text { Differences in opinion between clinical staff } \\
\text { concerning patient treatment }\end{array}$ & & & $2.25 \pm 1.08$ & $2.44 \pm 0.91$ & $2.22 \pm 1.02$ & 0.523 & 0.594 \\
\hline \multicolumn{9}{|c|}{ Workload } \\
\hline 20 & Amount of work assigned & $1.98 \pm 1.03$ & $2.19 \pm 0.94$ & $2.81 \pm 1.04$ & $2.53 \pm 1.13$ & $2.44 \pm 1.16$ & $\begin{array}{l}3.966 \\
3>4,5>2,1 \\
\end{array}$ & $0.004^{*}$ \\
\hline 21 & Full working day & $2.25 \pm 1.05$ & $2.66 \pm 0.98$ & $2.69 \pm 1.04$ & $2.67 \pm 1.17$ & $2.64 \pm 1.15$ & 1.541 & 0.192 \\
\hline 22 & Lack of time for relaxation & $2.36 \pm 1.06$ & $2.53 \pm 0.97$ & $2.72 \pm 1.09$ & $2.75 \pm 0.87$ & $2.78 \pm 1.17$ & 1.367 & 0.247 \\
\hline 23 & Lack of time to do assigned college work & $2.06 \pm 1.05$ & $2.43 \pm 1.01$ & $2.86 \pm 0.9$ & $2.89 \pm 0.89$ & $2.44 \pm 1.08$ & $\begin{array}{l}5.281 \\
4,3>2>5>1\end{array}$ & $0.000^{*}$ \\
\hline 24 & Late ending time & $2.25 \pm 1.14$ & $2.36 \pm 0.96$ & $2.56 \pm 1.05$ & $2.56 \pm 1.13$ & $2.56 \pm 1.18$ & 0.795 & 0.530 \\
\hline \multicolumn{9}{|c|}{ Patient treatment } \\
\hline 25 & $\begin{array}{l}\text { Lack of cooperation by patient in their home } \\
\text { care }\end{array}$ & & & $2.22 \pm 0.93$ & $2.78 \pm 0.83$ & $1.94 \pm 0.75$ & $\begin{array}{l}9.155 \\
4>3>5 \\
\end{array}$ & $0.000^{*}$ \\
\hline 26 & $\begin{array}{l}\text { Responsibilities for comprehensive patient } \\
\text { care }\end{array}$ & & & $2.11 \pm 0.85$ & $2.39 \pm 0.87$ & $2.06 \pm 0.79$ & 1.629 & 0.201 \\
\hline 27 & $\begin{array}{l}\text { Patients being late or not showing for their } \\
\text { appointments }\end{array}$ & & & $2.53 \pm 0.84$ & $2.94 \pm 0.83$ & $2.25 \pm 1.11$ & $\begin{array}{l}5.041 \\
4>3>5\end{array}$ & $0.008^{*}$ \\
\hline 28 & Working on patients with dirty mouths & & & $2.42 \pm 1.11$ & $2.83 \pm 1.08$ & $2.64 \pm 1.17$ & 1.244 & 0.292 \\
\hline \multicolumn{9}{|c|}{ Preclinical and clinical training } \\
\hline 29 & Difficulty in learning clinical procedures & & & $2.03 \pm 0.88$ & $2.06 \pm 0.92$ & $1.81 \pm 0.95$ & 0.802 & 0.451 \\
\hline 30 & $\begin{array}{l}\text { Difficulty in learning precision manual skills } \\
\text { required for preclinical and laboratory work }\end{array}$ & $1.81 \pm 0.94$ & $2.04 \pm 0.71$ & $1.86 \pm 0.87$ & $2.39 \pm 0.87$ & $2 \pm 0.86$ & $\begin{array}{l}2.803 \\
4>2,5>3,1\end{array}$ & $0.027^{*}$ \\
\hline \multicolumn{9}{|c|}{ Performance pressure } \\
\hline 31 & Competition with peers for grades & $2.25 \pm 1.07$ & $1.94 \pm 0.89$ & $2.33 \pm 0.99$ & $2.69 \pm 1.21$ & $2.06 \pm 1.15$ & $\begin{array}{l}3.063 \\
4>3,1>5,2 \\
\end{array}$ & $0.018^{*}$ \\
\hline 32 & Examination and grades & $2.36 \pm 1.11$ & $2.13 \pm 1.02$ & $2.56 \pm 1.05$ & $2.92 \pm 1.13$ & $2.25 \pm 1.18$ & $\begin{array}{l}3.160 \\
4>3>1>5>2\end{array}$ & $0.015^{*}$ \\
\hline \multicolumn{9}{|c|}{ Other } \\
\hline 33 & Relation with members of the opposite sex & $1.34 \pm 0.76$ & $1.49 \pm 0.93$ & $1.58 \pm 0.81$ & $1.78 \pm 1.1$ & $1.56 \pm 0.91$ & 1.341 & 0.256 \\
\hline 34 & $\begin{array}{l}\text { Difficult home/hostel environment in which to } \\
\text { study }\end{array}$ & $1.62 \pm 0.9$ & $1.87 \pm 0.9$ & $2.14 \pm 0.93$ & $2 \pm 1.04$ & $1.92 \pm 1.13$ & 1.718 & 0.147 \\
\hline 35 & Fear of unemployment after graduation & $1.98 \pm 1.1$ & $2.34 \pm 1.06$ & $2.94 \pm 1.15$ & $2.94 \pm 1.07$ & $2.69 \pm 1.24$ & $\begin{array}{l}6.258 \\
3,4,5>2>1\end{array}$ & $0.000^{*}$ \\
\hline 36 & Financial resources & $1.89 \pm 1.05$ & $1.92 \pm 0.92$ & $2.36 \pm 1.02$ & $2.22 \pm 1.17$ & $2.17 \pm 1.13$ & 1.610 & 0.173 \\
\hline 37 & Personal physical health & & & $1.86 \pm 0.72$ & $2.17 \pm 1.11$ & $1.94 \pm 1.09$ & 0.914 & 0.404 \\
\hline 38 & Availability of laboratory technicians & $1.51 \pm 0.93$ & $1.85 \pm 0.97$ & $2.17 \pm 0.97$ & $2.36 \pm 1.2$ & $2.03 \pm 1.06$ & $\begin{array}{l}4.533 \\
4,3,5>2,1\end{array}$ & $0.002^{*}$ \\
\hline
\end{tabular}

${ }^{*} \mathrm{p}<0.05$ consider statistically significant. One way ANOVA was appropriate. 
.The List of the 38 questions from the DES questionnaire is given in table7. The questions are divided into categories for ease of examination. Also the mean of each question is scored listed is a calculated mean illustrating how high the participants scored each question. Eighteen of the thirty-eight questions were had significant differences were compared across classes using Scheffe's test.

There are higher perception of stress among the first year of students than second year non-clinical students (Competition with peers for grades, $\mathrm{F}=3.063, \mathrm{p}=0.018$; Examination and grades, $\mathrm{F}=3.160, \mathrm{p}=0.015$ ), second year students had a higher perception of stress than their first year counterparts (Lack of time to do assigned college work, $\mathrm{F}=5.281, \mathrm{p}=0.00$; Difficulty in learning precision manual skills required for preclinical and laboratory work, $\mathrm{F}=2.803, \mathrm{p}=0.027$; Examination and grades, $\mathrm{F}=3.160, \mathrm{p}=0.015$ )

Similarly, final year students and interns had a higher perception of stress than their third year counterparts (Lack of confidence to be a successful dental student, $\mathrm{F}=2.518$, $\mathrm{p}=$ 0.042; Completing clinical requirements, $\mathrm{F}=55.026, \mathrm{p}=0.00$; Receiving criticism from supervisors about academic or clinical work, $F=7.26, p=0.00$; Attitudes of faculty towards women dental students, $\mathrm{F}=3.463, \mathrm{p}=0.009$; Lack of cooperation by patient in their home care, $\mathrm{F}=9.155, \mathrm{p}=0.00$; Patients being late or not showing for their appointments, $\mathrm{F}=5.041, \mathrm{p}=0.008$; Difficulty in learning precision manual skills required for preclinical and laboratory work, $\mathrm{F}=2.803$, $\mathrm{p}=0.027$; Competition with peers for grades, $\mathrm{F}=3.063$, $\mathrm{p}=0.018$; Examination and grades, $\mathrm{F}=3.160, \mathrm{p}=0.015$, Fear of unemployment after graduation, $\mathrm{F}=6.258, \mathrm{p}=0.00$; Availability of laboratory technicians, $\mathrm{F}=4.533, \mathrm{p}=0.002$ ). The perception of stress by third year students tended to be higher overall than for their fourth year counterparts. Analysis of variance showed significant differences in this direction on three DES questionnaire items (Amount of cheating in dental faculty, $\mathrm{F}=6.330, \mathrm{p}=0.00$; Expectation of dental faculty and what in reality it is like, $\mathrm{F}=3.936, \mathrm{p}=0.004$; Amount of work assigned, $\mathrm{F}=3.966, \mathrm{p}=0.004$ )

TABLE- 8 An overview of the top 6 stress factors is presented in table 8. The questions, with the highest mean were collected from table 7 and arranged in rows. Examination and grades in first year male and female, final year female, second year male $(2.81 \pm 1.17$ and $2.81 \pm 1.04,3.15 \pm 0.95,3.1 \pm$ 1.2 respectively), Fear of unemployment after graduation in males of second year and third year was $3.1 \pm 1.2$ and $3.64 \pm$

TABLE-8 The six highest stressors

\begin{tabular}{|c|c|c|c|c|c|c|c|c|c|}
\hline \multicolumn{10}{|l|}{ Male } \\
\hline \multicolumn{2}{|l|}{ I Year } & \multicolumn{2}{|l|}{ II Year } & \multicolumn{2}{|l|}{ III Year } & \multicolumn{2}{|l|}{ IV Year } & \multicolumn{2}{|l|}{ Intern } \\
\hline Q.no & $\begin{array}{l}\text { Mea } \\
n\end{array}$ & Q.no & Mean & Q.no & $\begin{array}{l}\text { Mea } \\
\mathrm{n}\end{array}$ & Q.no & $\begin{array}{l}\text { Mea } \\
\mathrm{n}\end{array}$ & Q.no & $\begin{array}{l}\text { Mea } \\
\mathrm{n}\end{array}$ \\
\hline $\begin{array}{l}\text { Financial } \\
\text { resources }\end{array}$ & $\begin{array}{l}2.19 \\
\pm \\
1.11\end{array}$ & $\begin{array}{l}\text { Lack of } \\
\text { confidence to be } \\
\text { a successful } \\
\text { dental student }\end{array}$ & $\begin{array}{l}2.6 \pm \\
1.07\end{array}$ & $\begin{array}{l}\text { Lack of time to } \\
\text { do assigned } \\
\text { college work }\end{array}$ & $\begin{array}{l}3 \pm \\
0.68\end{array}$ & $\begin{array}{l}\text { Fear of unemployment after } \\
\text { graduation }\end{array}$ & $\begin{array}{l}2.56 \\
\pm \\
1.13\end{array}$ & $\begin{array}{l}\text { Rules and regulations of } \\
\text { the faculty }\end{array}$ & $\begin{array}{l}2.2 \\
\pm \\
1.21\end{array}$ \\
\hline $\begin{array}{l}\text { Lack of time to } \\
\text { do assigned } \\
\text { college work }\end{array}$ & $\begin{array}{l}2.25 \\
\pm \\
1.06\end{array}$ & $\begin{array}{l}\text { Late ending } \\
\text { times }\end{array}$ & $\begin{array}{l}2.6 \pm \\
1.07\end{array}$ & $\begin{array}{l}\text { Fear of failing } \\
\text { a course or a } \\
\text { year }\end{array}$ & $\begin{array}{l}3 \pm \\
0.88\end{array}$ & $\begin{array}{l}\text { Lack of cooperation by patient } \\
\text { in their home care }\end{array}$ & $\begin{array}{l}2.67 \\
\pm 1 \\
\end{array}$ & Full working days & $\begin{array}{l}2.2 \\
\pm \\
1.21\end{array}$ \\
\hline $\begin{array}{l}\text { Lack of time } \\
\text { for relaxation }\end{array}$ & $\begin{array}{l}2.38 \\
\pm \\
1.09\end{array}$ & $\begin{array}{l}\text { Lack of time for } \\
\text { relaxation }\end{array}$ & $\begin{array}{l}2.7 \pm \\
0.95\end{array}$ & $\begin{array}{l}\text { Amount of } \\
\text { work assigned }\end{array}$ & $\begin{array}{l}3 \pm \\
0.88\end{array}$ & Lack of time for relaxation & $\begin{array}{l}2.67 \\
\pm \\
1.12\end{array}$ & $\begin{array}{l}\text { Working on patients with } \\
\text { dirty mouths }\end{array}$ & $\begin{array}{l}2.2 \\
\pm \\
1.26\end{array}$ \\
\hline $\begin{array}{l}\text { Late ending } \\
\text { times }\end{array}$ & $\begin{array}{l}2.38 \\
\pm \\
1.15\end{array}$ & Full working days & $\begin{array}{l}3 \pm \\
1.05\end{array}$ & $\begin{array}{l}\text { Full working } \\
\text { days }\end{array}$ & $\begin{array}{l}3 \pm \\
0.88\end{array}$ & $\begin{array}{l}\text { Difficulty in learning precision } \\
\text { manual skills required for } \\
\text { preclinical and laboratory work }\end{array}$ & $\begin{array}{l}2.67 \\
\pm \\
1.12\end{array}$ & $\begin{array}{l}\text { Fear of unemployment } \\
\text { after graduation }\end{array}$ & $\begin{array}{l}2.2 \\
\pm \\
1.37\end{array}$ \\
\hline $\begin{array}{l}\text { Competition } \\
\text { with peers for } \\
\text { grades }\end{array}$ & $\begin{array}{l}2.63 \\
\pm \\
1.2\end{array}$ & $\begin{array}{l}\text { Examination and } \\
\text { grades }\end{array}$ & $\begin{array}{l}3.1 \pm \\
1.2\end{array}$ & $\begin{array}{l}\text { Financial } \\
\text { resources }\end{array}$ & $\begin{array}{l}3 \pm \\
0.96\end{array}$ & $\begin{array}{l}\text { Patients being late or not } \\
\text { showing for their appointments }\end{array}$ & $\begin{array}{l}2.78 \\
\pm \\
0.83\end{array}$ & $\begin{array}{l}\text { Expectation of dental } \\
\text { faculty and what in } \\
\text { reality it is like }\end{array}$ & $\begin{array}{l}2.4 \\
\pm \\
0.63\end{array}$ \\
\hline $\begin{array}{l}\text { Examination } \\
\text { and grades }\end{array}$ & $\begin{array}{l}2.81 \\
\pm \\
1.17\end{array}$ & $\begin{array}{l}\text { Fear of } \\
\text { unemployment } \\
\text { after graduation }\end{array}$ & $\begin{array}{l}3.1 \pm \\
1.2\end{array}$ & $\begin{array}{l}\text { Fear of } \\
\text { unemploymen } \\
\text { t after } \\
\text { graduation }\end{array}$ & $\begin{array}{l}3.64 \\
\pm \\
0.63\end{array}$ & $\begin{array}{l}\text { Lack of time to do assigned } \\
\text { college work }\end{array}$ & $\begin{array}{l}3 \pm \\
1\end{array}$ & $\begin{array}{l}\text { Receiving criticism from } \\
\text { supervisors about } \\
\text { academic or clinical work }\end{array}$ & $\begin{array}{l}2.4 \\
\pm \\
1.18\end{array}$ \\
\hline \multicolumn{10}{|l|}{ Female } \\
\hline I Year & & II Year & & III Year & & IV Year & & Intern & \\
\hline Q.no & $\begin{array}{l}\text { Mea } \\
n\end{array}$ & Q.no & Mean & Q.no & $\begin{array}{l}\text { Mea } \\
\mathrm{n}\end{array}$ & Q.no & $\begin{array}{l}\text { Mea } \\
\mathrm{n}\end{array}$ & Q.no & $\begin{array}{l}\text { Mea } \\
\mathrm{n}\end{array}$ \\
\hline $\begin{array}{l}\text { Full working } \\
\text { days }\end{array}$ & $\begin{array}{l}2.19 \\
\pm \\
1.07\end{array}$ & $\begin{array}{l}\text { Amount of work } \\
\text { assigned }\end{array}$ & $\begin{array}{l}2.14 \\
\pm \\
0.91\end{array}$ & $\begin{array}{l}\text { Fear of } \\
\text { unemploymen } \\
\mathrm{t} \text { after } \\
\text { graduation }\end{array}$ & $\begin{array}{l}2.5 \\
\pm \\
1.19\end{array}$ & $\begin{array}{l}\text { Lack of time to do assigned } \\
\text { college work }\end{array}$ & $\begin{array}{l}2.85 \\
\pm \\
0.86\end{array}$ & Late ending times & $\begin{array}{l}2.86 \\
\pm \\
1.15\end{array}$ \\
\hline $\begin{array}{l}\text { Lack of time to } \\
\text { do assigned } \\
\text { college work }\end{array}$ & $\begin{array}{l}2.25 \\
\pm \\
1.04\end{array}$ & $\begin{array}{l}\text { Fear of } \\
\text { unemployment } \\
\text { after graduation }\end{array}$ & $\begin{array}{l}2.16 \\
\pm \\
0.95\end{array}$ & $\begin{array}{l}\text { Late ending } \\
\text { times }\end{array}$ & $\begin{array}{l}2.55 \\
\pm \\
1.06\end{array}$ & $\begin{array}{l}\text { Competition with peers for } \\
\text { grades }\end{array}$ & $\begin{array}{l}2.85 \\
\pm \\
1.13\end{array}$ & $\begin{array}{l}\text { Receiving criticism from } \\
\text { supervisors about } \\
\text { academic or clinical work }\end{array}$ & $\begin{array}{l}2.86 \\
\pm \\
1.2\end{array}$ \\
\hline $\begin{array}{l}\text { Lack of time } \\
\text { for relaxation }\end{array}$ & $\begin{array}{l}2.38 \\
\pm \\
1.06\end{array}$ & $\begin{array}{l}\text { Late ending } \\
\text { times }\end{array}$ & $\begin{array}{l}2.3 \pm \\
0.94\end{array}$ & $\begin{array}{l}\text { Amount of } \\
\text { cheating in } \\
\text { dental faculty }\end{array}$ & $\begin{array}{l}2.59 \\
\pm \\
1.14\end{array}$ & $\begin{array}{l}\text { Working on patients with dirty } \\
\text { mouths }\end{array}$ & $\begin{array}{l}2.96 \\
\pm \\
1.09\end{array}$ & Full working days & $\begin{array}{l}2.95 \\
\pm \\
1.02\end{array}$ \\
\hline $\begin{array}{l}\text { Late ending } \\
\text { times }\end{array}$ & $\begin{array}{l}2.38 \\
\pm \\
1.15\end{array}$ & $\begin{array}{l}\text { Lack of time to } \\
\text { do assigned } \\
\text { college work }\end{array}$ & $\begin{array}{l}2.42 \\
\pm \\
1.01\end{array}$ & $\begin{array}{l}\text { Lack of time } \\
\text { for relaxation }\end{array}$ & $\begin{array}{l}2.68 \\
\pm \\
1.09\end{array}$ & $\begin{array}{l}\text { Patients being late or not } \\
\text { showing for their appointments }\end{array}$ & $\begin{array}{l}3 \pm \\
0.83\end{array}$ & $\begin{array}{l}\text { Working on patients with } \\
\text { dirty mouths }\end{array}$ & $\begin{array}{l}2.95 \\
\pm \\
1.02\end{array}$ \\
\hline $\begin{array}{l}\text { Competition } \\
\text { with peers for } \\
\text { grades }\end{array}$ & $\begin{array}{l}2.63 \\
\pm \\
0.98\end{array}$ & $\begin{array}{l}\text { Lack of time for } \\
\text { relaxation }\end{array}$ & $\begin{array}{l}1.01 \\
2.49 \\
\pm \\
0.98\end{array}$ & $\begin{array}{l}\text { Amount of } \\
\text { work assigned }\end{array}$ & $\begin{array}{l}1.05 \\
2.68 \\
\pm \\
1.13\end{array}$ & $\begin{array}{l}\text { Fear of unemployment after } \\
\text { graduation }\end{array}$ & $\begin{array}{l}3.07 \\
\pm \\
1.04\end{array}$ & $\begin{array}{l}\text { Fear of unemployment } \\
\text { after graduation }\end{array}$ & $\begin{array}{l}3.05 \\
\pm \\
1.02\end{array}$ \\
\hline $\begin{array}{l}\text { Examination } \\
\text { and grades }\end{array}$ & $\begin{array}{l}2.81 \\
\pm \\
1.04\end{array}$ & Full working days & $\begin{array}{l}2.58 \\
\pm \\
0.96\end{array}$ & $\begin{array}{l}\text { Lack of time to } \\
\text { do assigned } \\
\text { college work }\end{array}$ & $\begin{array}{l}2.77 \\
\pm \\
1.02\end{array}$ & Examination and grades & $\begin{array}{l}3.15 \\
\pm \\
0.95\end{array}$ & $\begin{array}{l}\text { Lack of time for } \\
\text { relaxation }\end{array}$ & $\begin{array}{l}3.24 \\
\pm \\
0.89\end{array}$ \\
\hline
\end{tabular}


0.63 respectively, Full working day $2.58 \pm 0.96$ in second year female, Amount of work assigned in females of 3 rd year and interns were $2.68 \pm 1.13$ and $3.24 \pm 0.89$ respectively, Lack of time to do assigned college work $3 \pm 1$ in final year males, Receiving criticism from supervisors about academic or clinical work $2.4 \pm 1.18$ in male interns.

TABLE 9-Stress factors by year of study.

\begin{tabular}{|l|l|r|r|r|r|r|l|l|}
\hline S. no. & \multicolumn{1}{|c|}{ Factor } & First year & Second year & Third year & Fourth year & Intern & p-Value & Significance \\
\hline 1 & Selfefficacy beliefs & $1.8 \pm 0.56$ & $1.87 \pm 0.52$ & $2.02 \pm 0.59$ & $2.16 \pm 0.67$ & $1.98 \pm 0.7$ & 0.063 & NS \\
\hline 2 & $\begin{array}{l}\text { Faculty and } \\
\text { administration }\end{array}$ & $1.73 \pm 0.91$ & $1.73 \pm 0.65$ & $2.18 \pm 0.65$ & $2.37 \pm 0.76$ & $2.26 \pm 0.77$ & 0.000 & HS $^{*}$ \\
\hline 3 & Workload & $2.18 \pm 0.92$ & $2.43 \pm 0.8$ & $2.73 \pm 0.82$ & $2.68 \pm 0.89$ & $2.57 \pm 0.99$ & 0.024 & S $^{*}$ \\
\hline 4 & Patient treatment & & & $2.34 \pm 0.71$ & $2.75 \pm 0.73$ & $2.24 \pm 0.77$ & 0.009 & HS $^{*}$ \\
\hline 5 & $\begin{array}{l}\text { Preclinical and } \\
\text { clinical training }\end{array}$ & $1.81 \pm 0.94$ & $2.04 \pm 0.71$ & $1.94 \pm 0.83$ & $2.22 \pm 0.77$ & $1.9 \pm 0.84$ & 0.203 & NS \\
\hline 6 & $\begin{array}{l}\text { Performance } \\
\text { pressure }\end{array}$ & $2.3 \pm 1$ & $2.04 \pm 0.85$ & $2.44 \pm 0.94$ & $2.81 \pm 1.11$ & $2.15 \pm 1.09$ & 0.007 & HS $^{*}$ \\
\hline 7 & Personal factors & $1.67 \pm 0.71$ & $1.89 \pm 0.64$ & $2.17 \pm 0.66$ & $2.25 \pm 0.86$ & $2.06 \pm 0.81$ & 0.002 & HS $^{*}$ \\
\hline
\end{tabular}

$*_{\mathrm{p}}<0.05$ consider statistically significant. One way ANOVA was appropriate.

TABLE 9 The stress items were grouped into seven composite categories of related items. The mean stress score for each category was calculated by taking the average score of each item in the category. Ranking of the composite categories indicates that the category "faculty and administration" had the highest average mean stress score. "Personal factors" was the second highest ranking category with a mean stress score. Categories recording moderate mean stress scores were performance pressure, patient treatment and workload. There was highly significant and significant difference in mean score of stress factor of patients according to year of study except in Self-efficacy beliefs and Preclinical and clinical training, final year students perceived more stress than the other studying years, as there is a significant difference $(\mathrm{p}$ $<0.05$ ) in the mean scores of final year students for faculty administration, patient treatment, performance pressure and other personal problems.

Fig.2. Stress factors by gender and course of study

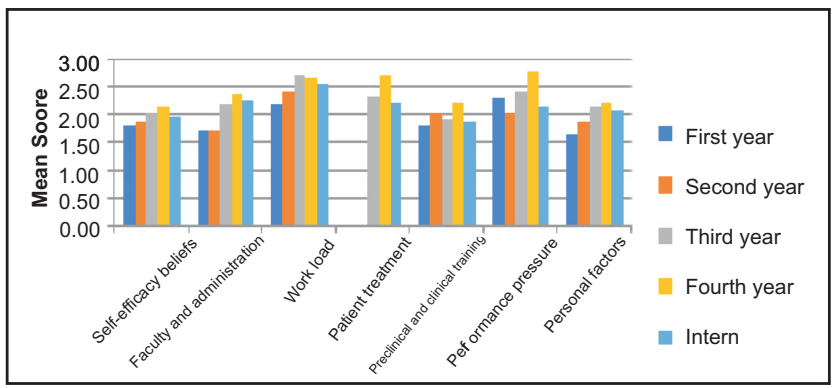

Fig.2. Stress factors by gender and course of study, selfefficacy beliefs (SEB); faculty and administration (FA); workload (WL); patient treatment (PT); preclinical and clinical training (PCT); performance pressure (PP); personal factors $(\mathrm{PF})$.

\section{Discussion:}

The present study was conducted to see the perceived sources of stress (PSS) amongst Indian dental students. Stress has been identified as a two-edged sword that either stimulates and motivates the students to peak performance or reduces the students a state of ineffectiveness. The professional educational process can be a stressful experience.[9] To become a responsible dental professional, students must acquire a high level of knowledge and work skills, as well as develop good attitudes towards patient care; all within a time period. Manivasakan S et al10 results confirmed that dental students experienced considerable stress in the clinical period of dental education as compared to medical students.

Fear of failing is significantly higher in 3rd year students in this study which may be attributed to the transition from preclinical to a clinical field whereas irreconcilable results were reported in other studies conducted in some other part of the world.[1,11,12]

"Fear of unable to catch up if getting behind the work" also reflected this difference between the classes in emphasis of their curriculum. Freshmen (first year) did not have enough experience to be too concern about learning clinical procedures as they were just beginners in learning, compared to sophomores (second year) who were more distressed by this item as they were just beginners to the clinical procedures. But seniors (third, final year and interns) ranked this item lower as they had experienced in handling clinical procedures. Similar finding were also found in studies conducted by Kumar S et al.[6]

The results of this study indicate that students were concerned with their relationships with the faculty and administration as well as their ability to grasp and absorb the amount of material presented to them.[13] The importance of good studentfaculty relationships and receiving good criticism from supervisors about academic or clinical work was evidenced by student rankings of this item as the most important stress area, concurrent findings which reported by various studies.[6,7,8] This is reflected in their ratings of the items referring to these areas and the composite ratings of stress categories of faculty and administration, patient treatment, performance pressure and personal factors. These composite categories were rated considerably higher than workload. The problems mentioned by students were poor student-faculty relationships, excessive pressures, amount of cheating in dental faculty, student favouritism and expectation of dental faculty. 
Faculty-related stress such as the amount of student cheating in the dental faculty, the atmosphere created by clinical supervisors and differences of opinion among clinical staff about patient treatment were significant findings in our research, as some faculty members could misuse their privilege and authority. In our perception of stress about faculty and administration, the fourth year students are more stressed than their third year counterparts as indicated by Newton JT et al[14]. The erratic feedback from faculty members was one of the primary stresses in the first year of dental school. However, there is a tendency in every school where some instructors behave in a professionally unacceptable manner. A certain amount of tension exists between students and faculty as a result of the faculty's evaluative and authoritative style.

Particularly, limitations of leisure time, examination anxiety and the transition stress caused by dealing with the clinical work are experienced as stressful. Dental school has rigorous curriculum that occupies most of the daytime hours of the students. Academic and clinical demands broaden the working hours of the students into the nights and weekends. As a result, students have little time to relax with their friends and might also face other potential sources of stress such as those involved in dealing with patients and acquiring relevant clinical skills.

In the present study some students are reported their personal problems as highly stressful such as marriage, alcohol addiction and drugs addiction, similar results were found in Garbee WH et al 8 study. These students have degrees of stress in academic and clinical areas.[15] In our study the amount of work assigned, mean stress scores showed steady increase through the year of study with a peak in the third year. The reason for such finding may be the transition from preclinical to a clinical procedure and which was contrary to Sofola $\mathrm{OO}$ et al.[16]

The highest stressors reported by both third and fourth year dental students were clinical and patient requirements. This finding is supported by Al-Sowygh ZH et al[17] which also indicated that clinical training issues in dental students impose a highest level of stress.[7] This stress could be due to fact that dental students are required to complete a certain quota of cases to be eligible to appear in the final examination. Competition with peers for examination and grades in the third year produced considerable stress. In this respect, parallel studies with similar findings were also seen in other dental schools.[3,6] Considering the large quantities of difficult material that students are required to master, it is not surprising that students often consider themselves inadequate for the task. Grades and marks in addition to academic achievement can also be used as indicators of stress, where stressed students may have low grades and low achievement. Among the years of study, fourth year students had the highest scores for PSS, followed by third-year students. First-year students had the lowest score. Similar finding were reported by Al-Sowygh ZH[7]. Fourth year students feared the reaction of their parents to failure[18] and also had a fear of not qualifying for postgraduate studies[19], doubting whether they had the capability to be admitted in their desired postgraduate programme.

Financial resource was the another aspect about which male students were more concerned than females which was in contrast to a study conducted by Heath JR et al,1where no such gender difference was observed, whereas a Musser LA et al20 reported a gender difference for financial concern. Scores for 'financial problems' tended to increase progressively with the passage of time, peaking at a mean of in the third year, perhaps indicating the accumulation of debt, whereas irreconcilable results were shown in Heath JR et al.[1]

Fear of unemployment after graduation has been the sixth highest stressor for all years and in both sexes; the explanation may be the current trend in dental education in India, where about 25,000 dentists pass out of 310 dental institutions every year.[15] Although the current oral health situation in India has tremendous unmet medical needs, employment opportunities are limited, as there is no oral health policy at the national level in India, and there are no dental services provided by the public health sector in many states. Accordingly, job opportunities for fresh undergraduates are concentrated primarily in the private sector where there is increased competition that would restrict their income.

The most important limitation of this study is its cross? sectional design. Longitudinal research could be helpful to investigate the amount of stress (increase or decrease) amongst students during their academic career. Further research could be conducted in different dental education phases in different dental colleges to determine the generalizability of these findings. There is a lack of information concerning the stress level of students before they enter the dental education programme. Bias cannot be ruled out because information was collected from selfadministered questionnaires. 


\section{Summary:}

The purpose of this study was to access the perceived sources of stress amongst undergraduate dental students in Dental College located in Bareilly. Dental students displayed relatively high perceived stress scores, male students perceived more stress than did females, married compared with single students reported more stress. We found the significant values of self-efficacy, workload, behavioral disengagement, positive reframing and denial to independently affect the perceived stress score. Students have little time to relax with their friends, the amount of work assigned, mean stress scores showed steady increase through the year of study with a peak in the third year. The highest stressors reported by both third and fourth year dental students were clinical and patient requirements. Fear of unemployment after graduation has been the sixth highest stressor for all years and in both sexes.

The problems mentioned by students were poor studentfaculty relationships, excessive pressures, amount of cheating in dental faculty, student favouritism and expectation of dental faculty. In our perception of stress about faculty and administration, the fourth year students are more stressed than their third year. Students were concerned about how they are treated by the faculty. Faculty are frequently hard on them when trying to motivate them for better performance. Possibly, better ways of motivating students could be methods that would not create such high degrees of stress. Students need to be treated as professionals and ways to accomplish this need to be investigated. Students were concerned about the academic pressures of dental school whereas rigorous training in dental school is necessary to prepare students. Students react in different ways to stress in varying intensity. Those who feel greater stress, require more assistance in handling this stress. The types of supports or services that are required need to be identified and provided for student assistance.

\section{Conclusion:}

The primary sources of stress as perceived by dental student were examinations and grades, full working day and receiving criticism from supervisors about academic or clinical work. Our study expressed higher levels of stress in undergraduate, hence students should be taught positive coping strategies and various stress managing techniques to improve the ability to cope with the demanding professional course. Moreover, there was a significant difference in perception of stress for performance pressure and personal factors between the years of study. It appears there is a need for the establishment of student advisors and counsellors combined with a faculty advising system in addition to student-oriented programmes.

\section{References:}

1. Heath JR, Macfarlane TV, Umar MS. Perceived sources of stress in dental students. Dental Update 1999:26(3):94-8.

2. Humphris G, Blinkhorn A, Freeman R, Gorter R, Reddick GH, Murtomaa $\mathrm{H}$, et al. Psychological stress in undergraduate dental students: baseline results from seven European dental schools. Eur J Dent Educ 2002;6(1):22-9.

3. Kirwan M, Armstrong D. Investigation of burnout in a sample of British general practitioners. British Journal of General Practice 1995;45(1):259-60.

4. Basudan S, Binanzan N, Alhassan A. Depression, anxiety and stress in dental students. International Journal of Medical Education 2017;8(4):179-86

5. Alzahem AM, Molen HTV, Alaujan AH, Schmidt HG, Zamakhshary MH. Stress amongst dental students: a systematic review. Eur J Dent Educ 2011;15(5):8-18

6. Kumar S, Dagli RJ, Mathur A, Jain M, Prabu D, Kulkarni S. Perceived sources of stress amongst Indian dental students. Eur J Dent Educ 2009;13(1):39-45.

7. Al-Sowygh ZH. Academic distress, perceived stress and coping strategies among dental students in Saudi Arabia. The Saudi Dental Journal 2013;25(3):97-105.

8. Garbee WH, Zucker SB, Selby GR. Perceived sources of stress among dental students JADA 1980;100(1):853-7.

9. Sugiura G, Shinada K, Kawaguchi Y. Psychological well-being and perceptions of stress amongst Japanese dental students. Eur J Dent Educ 2005;9(2):17-25.

10. Manivasakan S, Raman SK, Devy AS, Saravanakumar R. The clinical dental undergraduate's perception of stress origin in India: A crosssectional study. J Nat Sc Biol Med 2018;9(2):197-200.

11. Muirhead V, Locker D. Canadian dental students' perceptions of stress. J Can Dent Assoc 2007;73(7):323-7.

12. Murphy RJ, Gray SA, Sterling G, Reeves K, DuCette J. A comparative study of professional student stress. J Dent Educ 2009;73(6):328?37.

13. Al-Saleh SA, Al-Madi EM, Al-Angari NS, Al-Shehri HA, Shukri M. Survey of perceived stress-inducing problems among dental students, Saudi Arabia. The Saudi Dental Journal 2010;22(3):83-8.

14. Newton JT, Baghaienaini F, Goodwin SR, Invest J, Lubbock M, Saghakhaneh NM. Stress in dental schools: a survey of students. Dent Update 1994;21(3):162-4.

15. Yadav $\mathrm{S}$, Rawal G. The current status of dental graduates in India. Pan Afr Med J 2016;23(4):22-5.

16. Sofola OO, Jeboda SO. Perceived sources of stress in Nigerian dental students. Eur J Dent Educ 2006;10(6):20-3.

17. Sekhon TS, Grewal S, Gambhir RS, Sharma S. Perceived sources of stress among dental college students: An Indian perspective. European Journal of General Dentistry 2015;4(3):121-6.

18. Deb S, Strodl E, Sun J. Academic Stress, Parental Pressure, Anxiety and Mental Health. International Journal of Psychology and Behavioral Sciences 2015;5(1):26-34.

19. Acharya S. Factors affecting stress among Indian dental students. J Dent Educ 2003: 67(4):1140-8

20. Musser LA, Lloyd C. The relationship of marital status and living arrangement to stress among dental students. J Dent Educ 1985;49(5):573-8 